\title{
Poorly differentiated pulmonary synovial sarcoma with SYT gene amplification: A case report
}

\author{
XIAOYAN LI* ${ }^{*}$ DAN WU*, YUHUI ZHENG, HUANXING YANG and YINGHONG YANG \\ Department of Pathology, Fujian Medical University Union Hospital, Fuzhou, Fujian 350001, P.R. China
}

Received September 7, 2018; Accepted November 21, 2018

DOI: $10.3892 / \mathrm{mco} .2018 .1787$

\begin{abstract}
Fluorescence in situ hybridization (FISH) and reverse-transcription polymerase chain reaction (RT-PCR) analysis may be used for the diagnosis of synovial sarcoma (SS), particularly of the poorly differentiated type. While the majority of the studies report that the SYT FISH probe is considered to be break-apart in SS, with two orange and two green signals, the SYT probe in the present case of a 52-year-old male patient with pulmonary SS displayed orange and green signal separation, along with SYT orange signal amplification. RT-PCR was used to verify that the SYT gene amplification was another form of expression of SYT-SSX gene fusion $\mathrm{t}(\mathrm{X} ; 18)(\mathrm{p} 11 ; \mathrm{q} 11)$. In this case, the tumour sample obtained by biopsy was small; therefore, the definitive diagnosis of poorly differentiated SS originating from the lung with SYT gene amplification was confirmed by FISH and RT-PCR. Therefore, these mature biomarkers, which are available as immunohistochemical stains in the molecular pathology laboratory, may help pathologists to diagnose intractable soft tissue tumours based only on small cytological specimens.
\end{abstract}

\section{Introduction}

Synovial sarcoma (SS) may originate from pluripotent stem cells of unknown origin. SS is a malignant tumour bearing the characteristics of diphasic epithelial and mesenchymal cell differentiation. SS has three histological types, including monophasic, diphasic and poorly differentiated. The monophasic and poorly differentiated types are difficult to diagnose based on histomorphology alone, and molecular detection methods play an important accessory diagnostic role in such cases. SS has a characteristic chromosome translocation $\mathrm{t}(\mathrm{X} ; 18)(\mathrm{p} 11 ; \mathrm{q} 11)$,

Correspondence to: Dr YingHong Yang, Department of Pathology, Fujian Medical University Union Hospital, 29th Xinquan Road, Fuzhou, Fujian 350001, P.R. China

E-mail: yyh1555@163.com

${ }^{*}$ Contributed equally

Key words: synovial sarcoma, lung, SYT gene, fluorescence in situ hybridization, reverse transcription-polymerase chain reaction resulting in SYT-SSX gene fusion. This genovariation may be detected by fluorescence in situ hybridization (FISH) and reverse transcription-polymerase chain reaction (RT-PCR) analysis. The majority of studies have reported that the SYT FISH probe was considered to be break-apart when a pair of orange and green signals was separated by a distance greater than the size of one hybridization signal in the SS cell nucleus (1). In the present case report, the SYT FISH probe displayed orange and green signal separation along with the SYT orange signal amplification in a poorly differentiated pulmonary SS.

\section{Case report}

A 52-year-old male patient with a smoking history of 2 packs/day for $>30$ years presented with left-sided chest pain for 2 months. The patient had no cough, sputum, dyspnoea or palpitations, but reported a $5-\mathrm{kg}$ weight loss since the appearance of the symptoms. Thoracic pre- and post-contrast enhanced computed tomography (CT) scans revealed a solid mass with rough edges in the upper lobe of the left lung, $8.1 \times 8 \mathrm{~cm}$ in size. In addition, another small nodule with greatest diameter $0.5 \mathrm{~cm}$ displaying the same characteristics was identified in the inferior lobe of the right lung, and multiple enlarged lymph nodes in the left pulmonary hilum and mediastinum were also observed. Magnetic resonance imaging examination revealed multiple disseminated nodules in the bilateral frontal and parietal lobes, and color Doppler flow imaging also demonstrated bilateral supraclavicular lymph node enlargement. The patient underwent CT-guided percutaneous transthoracic needle aspiration biopsy of the mass in order to determine the clinicopathological diagnosis.

The tissue sample was fixed in formalin and embedded in paraffin, then processed according to the standard procedure including dehydration, clearing, wax embedding and sectioning, and stained with hematoxylin and eosin. Immunohistochemical (IHC) staining for vimentin, epithelial membrane antigen (EMA), CD34, thyroid transcription factor 1 (TTF-1), cytokeratin (CK), calretinin, Wilms' tumor-1 (WT-1), mesothelial cell (MC), napsin A, surfactant protein (SP)-B, P40, P63, CK5/6, CD56, synaptophysin (Syn), chromogranin A (CgA), desmin, CD99, S-100, CD45, CDX2, placental alkaline phosphatase (PLAP), CK19, glypican-3 and Ki-67 (Fuzhou Maixin Biotech Co., Ltd., Fuzhou, China) was performed in the Lumatas automated immunostainer (Fuzhou Maixin Biotech Co., Ltd.) with appropriate controls. 
For FISH, 2.5- $\mu \mathrm{m}$ paraffin-embedded sections were transferred to APES and baked for $2 \mathrm{~h}$ at $65^{\circ} \mathrm{C}$. The slides were deparaffinized by xylene and washed in $100 \%$ ethanol. Following pretreatment in boiling water for $20 \mathrm{~min}$, the slides were incubated in $0.5 \mathrm{mg} / \mathrm{ml}$ pepsin solution (in $0.01 \mathrm{~N} \mathrm{HCl}$, $\mathrm{pH} 2.0$ ) at $37^{\circ} \mathrm{C}$ for $7 \mathrm{~min}$. Then, the SYT break-apart probe mixture (probe:hybridization buffer:purified $\mathrm{H}_{2} \mathrm{O}=1: 7: 2$, GP Medical Technologies Co., Ltd., Beijing, China) was added to the slides in the dark. The slides were then incubated at $83^{\circ} \mathrm{C}$ for $5 \mathrm{~min}$ to co-denature, followed by hybridization with the probes in StatSpin ThermoBrite (Abbott Pharmaceutical Co. Ltd., Lake Bluff, IL, USA) at $42^{\circ} \mathrm{C}$ overnight. Following hybridization, the slides were counterstained with $10 \mu \mathrm{l}$ DAPI reagent (Vysis, Abbott Laboratories, Abbott Park, IL, USA) and coverslipped. Fluorescence signals were observed by a fluorescence microscope (Olympus BX51; Olympus Corporation, Tokyo, Japan).

For RT-PCR analysis, total RNA was isolated from paraffin-embedded tumour tissue sections according to the nucleic acid extraction kit protocol (Amoydx Biomedical Technology, Xiamen, China) and reverse-transcribed with Prime Script ${ }^{\mathrm{TM}}$ RT reagent kit (Takara Biotechnology Co., Ltd., Dalian, China). The tumour cDNA was amplified with primers for the SYT-SSX gene (forward primer for SYT: 5'-CCAGCAGAGGCCTTATGGATA-3'; reverse primer for SXX1: 5'-GTGCAGTTGTTTCCCATCG-3' $118 \mathrm{bp}$, and for SXX2: 5'-GCACAGCTCTTTCCCATCA-3', 118 bp). The h-actin gene (forward primer: 5'-TGGGTGTGAACCATG AGAAGT-3' and reverse primer: 5'-AAGGCCATGCCAGTG AGCTT-3' 310 bp) was used as an internal control for PCR and for quality assessment of the tumour cDNA. The amplification conditions included an initial denaturation step at $95^{\circ} \mathrm{C}$ for $5 \mathrm{~min}$, followed by denaturation at $95^{\circ} \mathrm{C}$ for $30 \mathrm{sec}$, annealing at $55^{\circ} \mathrm{C}$ for $30 \mathrm{sec}$, and extension at $72^{\circ} \mathrm{C}$ for $30 \mathrm{sec}$ for 40 cycles, with a final extension step at $72^{\circ} \mathrm{C}$ for $10 \mathrm{~min}$. The PCR products were separated by electrophoresis in agarose gels and visualized with ethidium bromide. The amplified fragments were identified according to their size on gels.

On histological examination of the tissue sections under an optical microscope, the boundary between the tumour and the surrounding lung tissue was unclear. The tumour cells around the thin-walled vessels in the stroma appeared to be loosely attached to each other and they were small and round, with scant cytoplasm and round or oval cell nuclei with homogeneous granulated chromatin. A small nucleolus and pathological mitoses were readily identified (Fig. 1A).

On IHC examination, the tumour cells were diffusely positive for vimentin (Fig. 1B) and EMA (Fig. 1C), focally positive for CD34 and TTF-1, and 80\% were Ki-67-positive (Fig. 1D). The tumour cells were negative for CK, calretinin, WT-1, MC, napsin A, SP-B, P40, P63, CK5/6, CD56, Syn, CgA, desmin, CD99, S-100, CD45, CDX2, PLAP, CK19 and glypican-3 expression.

As regards FISH, the SYT probe was considered to be break-apart when a pair of orange and green signals was separated by a distance greater than the diameter of one hybridization signal. Tumour cells positive for SYT exhibited at least one pair of break-apart signals per nucleus. In this case, five different tumour areas were observed under the fluorescence microscope with clear DAPI counterstaining and fluorescence signals. In each tumour zone, 20 non-overlapping cell nuclei with SYT signal were continuously counted. The total ratio of SYT-positive cells was calculated as $67 \%$ in 100 cells. Interestingly, $60 \%$ of the cell nuclei presented as clusters of orange signals ( $\sim 10-12$ signals per nucleus) against the background of separated signals, while the green signals were $\sim 1-3$ per nucleus (Fig. 1E).

In addition, RT-PCR was applied to confirm the FISH results and SYT-SSX2 gene fusion transcripts were detected by electrophoresis in agarose gels (Fig. 1F).

The final diagnosis was 'small round-cell malignant nodules in the upper lobe of the left lung, with molecular pathological characteristics consistent with poorly differentiated SS'.

\section{Discussion}

In the present case, the examination of the lung mass biopsy specimens under the microscope revealed small round-cell malignant nodules. The histological and immunohistochemical findings did not include diagnostic pathological characteristics. Lung adenocarcinoma, squamous cell carcinoma, small-cell carcinoma, as well as mesothelioma, lymphoma, and other common types of poorly differentiated carcinomas that commonly metastasize to the lung should be ruled out. However, depending on the above-mentioned evidence, which should always lead to diagnostic hesitancy, it is important to make the differential diagnosis clearly because the presence of overlapping categories which have significant prognostic and therapeutic implications respectively. In the present case, the tumour did not display specific epithelial and/or mesenchymal morphological characteristics; therefore, it was difficult to diagnose poorly differentiated SS based only on a small number of tumour cells. The morphological characteristic of SS, consisting of with small round cells, markedly overlapped with other sarcomas, including Ewing's sarcoma/peripheral neuroectodermal tumour (ES/PNET) family, neuroblastoma, rhabdomyosarcoma, malignant hemangiopericytoma and mesenchymal chondrosarcoma. The immunohistochemical phenotype was not helpful for the diagnosis of SS, with vimentin- and EMA-positive results.

The chromosomal translocation $\mathrm{t}(\mathrm{X} ; 18)(\mathrm{p} 11.2 ; \mathrm{q} 11.2)$ is both sensitive and specific for SS. Use of this chromosomal anomaly as a diagnostic biomarker may enable accurate diagnosis of SS, particularly when the available specimens are small. In our case, the balanced chromosomal translocation was detected by both FISH and RT-PCR. In several laboratories, FISH is performed to confirm the diagnosis of SS as the preferred molecular test; it allows the detection of molecular characteristics together with histomorphology, and non-neoplastic areas can be excluded (2). Under the fluorescence microscope, 5 tumour areas and a total of 100 tumour nuclei were counted, and the positive ratio of SYT gene break-apart was found to be $67 \%$. Surprisingly, $60 \%$ tumour nuclei presented SYT $\mathrm{C}^{\prime}$-terminal fragment amplification (orange spectrum $800 \mathrm{~kb}$, green spectrum $1,000 \mathrm{~kb}$ ) in the genetic disruption background. In order to explore whether the SYT amplification was another expression form of the SYT-SSX gene fusion, RT-PCR was used to verify the results, and SYT-SSX2 gene fusion transcripts were identified in the agarose gels. Combined with the FISH and PCR results, there was sufficient evidence to support the diagnosis of poorly differentiated SS in the upper lobe of the left lung. 
A

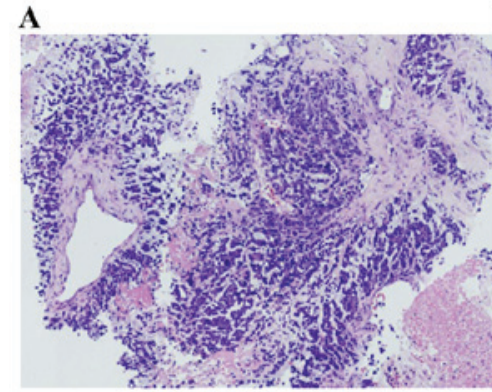

B

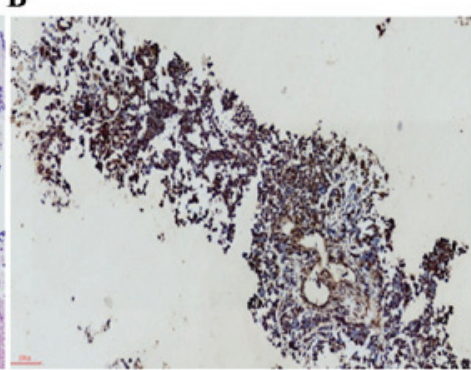

D

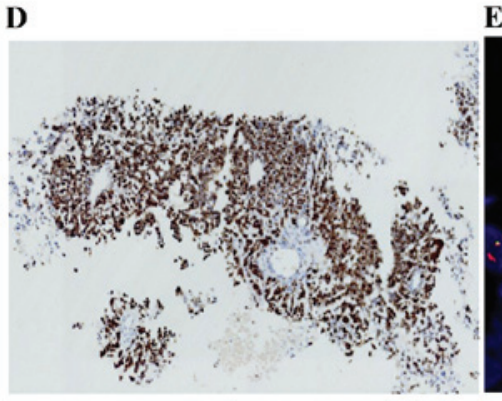

$\mathbf{E}$

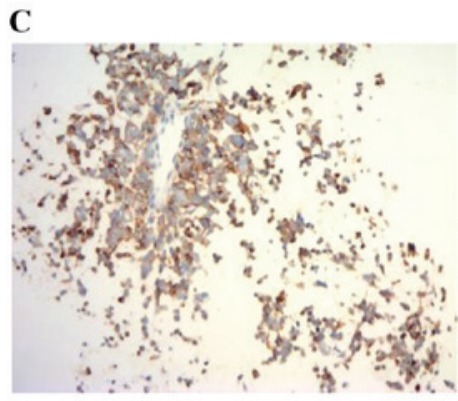

F

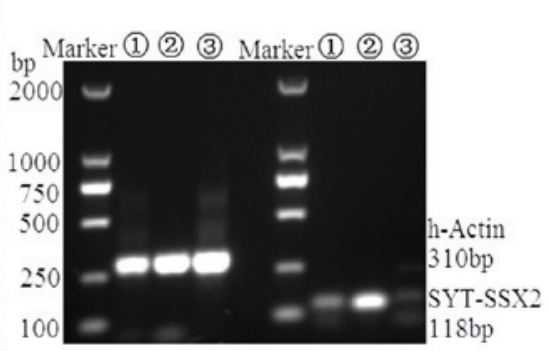

Figure 1. Histomorphology, immunochemistry, FISH and RT-PCR results of poorly differentiated synovial sarcoma. (A) The tumour cells around the thin-walled vessels were small and round, with scant cytoplasm and round or oval nuclei; haematoxylin and eosin stain, magnification, x100. (B) Immunostaining for vimentin was diffusely positive; magnification, x100. (C) Immunostaining for epithelial membrane antigen was diffusely positive; magnification, x200. (D) Immunostaining for Ki-67 was positive (80\%); magnification, x100. (E) The FISH images revealed $60 \%$ of tumour cell nuclei forming clusters of orange signals gathered against the background of the orange and green signal break-apart. (F) The RT-PCR result revealed the SYT-SSX2 fusion transcripts in agarose gels: (1)(3) present case, (2) positive control. FISH, fluorescence in situ hybridization; RT-PCR, reverse transcription-polymerase chain reaction.

According to the FISH product specification, the C'-terminal of the SYT gene (orange fluorescence) is the transcriptional activation domain, which is rich in glutamine, proline, glycine and tyrosine. In the present case, multiple orange signals in clumps were detected in over half of the tumour cells. In view of this observation, it was hypothesized that translocation may activate the SYT transcriptional activation domain resulting in the amplification of the functional gene fragment. Then, the SYT-SSX fusion protein may cause a disorder of gene expression in tumour cells (3). Some studies have confirmed that the genes involved in the Wnt signalling pathway, including TLE1, were upregulated by the SYT-SSX fusion proteins $(4,5)$, promoting the malignant progression of SS.

Some researchers have reported that the SYT-SSX fusion transcripts are not only a definitive diagnostic marker of SS, but also yield important independent prognostic information (6). It was reported that a patient with a SS bearing the SYT-SSX2 translocation had a better prognosis compared with a patient bearing the SYT-SSX1 translocation $(7,8)$. This conclusion was supported by a multi-institutional retrospective study of 243 SS patients by Ladanyi et al (9). The patient in the present case harboured the SYT-SSX2 fusion transcript, as shown by RT-PCR, and exhibited no other symptoms apart from emaciation during the 6-month interval from the date of symptom onset to the date of death in August, 2017, although multiple lesions were detected in the lungs, brain and lymph nodes of the mediastinum, left pulmonary hilum and supraclavicular regions when the patient first visited a doctor on February 2017. During those 6 months, the patient had been taking traditional Chinese medicines at home, having declined radiotherapy and chemotherapy.

In summary, this case of poorly differentiated SS originating from the lung with SYT gene amplification was confirmed by
FISH and RT-PCR. These mature biomarkers, available as immunohistochemical stains in the molecular pathology laboratory, may help pathologists to diagnose intractable soft tissue tumours based only on small cytological specimens (10).

\section{Acknowledgements}

The authors would like to thank the staff in the Pathology Department of Fujian Medical University Union Hospital.

\section{Funding}

No funding was received.

\section{Availability of data and materials}

The data and materials are available from the corresponding author on reasonable request.

\section{Authors' contributions}

$\mathrm{XL}$ is the major contributor to the writing of the manuscript. DW collected all the patient's clinical and follow-up visit data. $\mathrm{XL}$ and DW are co-first authors. HY performed the histological examination. YZ performed the molecular examination. YY provided the tissue materials and pathological data. All the authors have approved the final version of the manuscript.

\section{Ethics approval and consent to participate}

The study was approved by the Institute Research Ethics Committee of Fujian Medical University Union Hospital. 


\section{Patient consent for publication}

Informed consent for publication of the case details and any associated images was obtained from the patient's family according to the regulations of Institute Research Ethics Committee of Fujian Medical University Union Hospital.

\section{Competing interests}

The authors declare that they have no competing interests.

\section{References}

1. Sun B, Sun Y, Wang J,Zhao X,Zhang S,Liu Y,Li X,Feng Y,Zhou H and Hao X: The diagnostic value of SYT-SSX detected by reverse transcriptase-polymerase chain reaction (RT-PCR) and fluorescence in situ hybridization (FISH) for synovial sarcoma: A review and prospective study of 255 cases. Cancer Sci 99: 1355-1361, 2008.

2. Pyden AD and Lin X: Synovial sarcoma presenting as a lung mass and diagnosed by cytology. Diagn Cytopathol 44: 434-437, 2016.

3. dos Santos NR, de Bruijn DR, Kater-Baats E, Otte AP and van Kessel AG: Delineation of the protein domains responsible for SYT, SSX and SYT-SSX nuclear localization. Exp Cell Res 256: 192-202, 2000.
4. Nagayama S, Katagiri T, Tsunoda T, Hosaka T, Nakashima Y, Araki N, Kusuzaki K, Nakayama T, Tsuboyama T, Nakamura T, et al: Genome-wide analysis of gene expression in synovial sarcomas using a cDNA microarray. Cancer Res 62: 5859-5866, 2002.

5. Baird K, Davis S, Antonescu CR, Harper UL, Walker RL, Chen Y, Glatfelter AA, Duray PH and Meltzer PS: Gene expression profiling of human sarcomas: Insights into sarcoma biology. Cancer Res 65: 9226-9235, 2005.

6. Sun Y, Sun B, Wang J, Cai W, Zhao X, Zhang S and Hao X: Prognostic implication of SYT-SSX fusion type and clinicopathological parameters for tumor-related death, recurrence and metastasis in synovial sarcoma. Cancer Sci 100: 1018-1025, 2009.

7. Thway K and Fisher C: Synovial sarcoma: Defining features and diagnostic evolution. Ann Diagn Pathol 18: 369-380, 2014.

8. Maleki S, Cajigas A, Moss J, Ramesh KH and Khader S: Fine-needle aspiration biopsy of prostate synovial sarcoma: A case report and review of the literature. Diagn Cytopathol 45: 168-172, 2017.

9. Ladanyi M, Antonescu CR, Leung DH, Woodruff JM, Kawai A, Healey JH, Brennan MF, Bridge JA, Neff JR, Barr FG, et al: Impact of SYT-SSX fusion type on the clinical behavior of synovial sarcoma: A multi-institutional retrospective study of 243 patients. Cancer Res 62: 135-140, 2002.

10. Dodd LG, Ware P, Duncan D and Hertel J: Doing more with less: New markers for sarcoma diagnosis and their applicability to cytology specimens. Diagn Cytopathol 44: 351-360, 2016. 